\title{
Development of a measure of social inclusion for arts and mental health project participants
}

\section{Abstract \\ Background}

Promoting the social inclusion of mental health service users is a UK policy priority, but the development of outcome measures in this area is at an early stage

Aim

To develop a social inclusion measure for use in a study assessing the outcomes of arts participation for people with mental health needs.

\section{Method}

Concept and question development based on literature review, national and European surveys and results of a survey of arts and mental health projects. Measure piloted with 23 arts participants/service user researchers and field tested with 88 arts project participants returning questionnaires including the social inclusion measure, a measure of empowerment and the CORE mental health measure.

\section{Results}

Three scales were constructed measuring social acceptance, social isolation and social relations. Internal consistency was good for the individual scales and for the measure as a whole. Correlations with empowerment and CORE scores indicate reasonable predictive power for the population.

\section{Conclusions}

Tests to date indicate the measure is acceptable and measures relevant concepts with good internal consistency. Test-retest reliability and construct validity are not established and replication is required to confirm internal consistency and establish a normative profile for the population.

\section{Declaration of interest}

The research was jointly commissioned and funded by the Department for Culture, Media \& Sport and the Department of Health. The views expressed are the authors' and do not necessarily reflect those of the funders. 


\section{Introduction}

In 2004, the Social Exclusion Unit (SEU), located within the UK's Office of the Deputy Prime Minister (ODPM), published a report addressing social exclusion and mental health. The report identified the causes of exclusion as lying in large part in the stigmatisation of mental ill health and in a focus on medical symptoms at the expense of enabling people to participate in their local communities (ODPM, 2004). Over a third of respondents to the SEU's consultation identified access to recreational activities, including participation in the arts, as essential to promote social inclusion, and promoting access to arts opportunities was a key recommendation of the report. However, it was acknowledged that the evidence base for arts participation is currently weak. As part of a 27-point action plan the Department for Culture Media and Sport, in partnership with the Department of Health, therefore commissioned a study to contribute to the evidence base regarding the mental health benefits and social inclusion outcomes of participation in arts projects. This paper reports the work carried out to develop a measure of social inclusion for the study.

A review of art for health activity undertaken for the Health Development Agency (2000) indicated that arts participation may have health-related benefits such as increased self-esteem and self-determination. In addition, small-scale studies have reported specific benefits for people with mental health problems including fewer hospital re-admissions (Colgan et al., 1991), lower levels of depression (Huxley, 1997) and reduced rates of GP consultation (Everitt \& Hamilton, 2003). However, no studies assessing the impact of arts participation on social inclusion appeared to have been carried out and no published measure of social inclusion appeared to be available, although an untested measure developed in collaboration with service users has since been published (Stickly \& Shaw, 2006). It was therefore necessary to develop a new instrument for use in our study.

\section{Method}

\section{Concept and question development}

Understanding of the concept of social inclusion and its relationship to concepts such as social exclusion, social capital and social networks is at an early stage (McKenzie et al., 2002) and the initial work undertaken in developing a new instrument was therefore wide ranging. Since it was not feasible to systematically search all the relevant literatures within the study timescale, key texts cited in the SEU report, identified through comprehensive reviews carried out for that report, were used as a basis for a review of published literature. Further published and unpublished literature, including service user contributions, was identified via the websites of the major national mental health charities. In addition, a survey of arts and mental health projects in England, carried out for the first phase of our study, included questions about current evaluation practice and projects were asked to provide copies of any instruments they used for evaluation. These were searched for questions relevant to the concepts emerging from the literature review. Published art-in-health research reports and the national labour force and household surveys carried out by the Office for National Statistics were also searched for relevant questions, as were European sources such as the European Commission's Eurobarometer. See Hacking et al. (2006) for further details of the literature review and survey. 
Concepts derived from the published and unpublished literature were categorised and mapped against questions already in use at arts and mental health projects or reported elsewhere. These included: bonding and bridging social capital (Putnam, 1995); social acceptance, neighbourhood cohesion and engagement in leisure and cultural activity (Gallie \& Paugham, 2002); citizenship (Matarasso, 1997); and perceived security of housing tenure (Bates, 2005).

Where previously used questions did not relate well to our participant group, we adapted them for the measure. The 22 resulting questions were grouped in six categories relating to: building social capital (6 items); social acceptance (5 items); neighbourhood cohesion ( 2 items); security of housing tenure ( 2 items); engagement in leisure and cultural activities ( 3 items); and citizenship (4 items). Because different formats and rating scales had been used by the different sources from which they were drawn, all items were framed as Likert-type statements with a four-point frequency scale for responses ('not at all', 'sometimes', 'often', 'most/all of the time'). Occupational activity, which is widely regarded as a strong indicator of social inclusion, was measured by separate questions derived from the Labour Force Survey (www.statistics.gov.uk/STATBASE/Source.asp?vlnk=358).

\section{Piloting}

The measure was piloted with 15 participants at two arts and mental health projects and eight members of a service user research group. Views were sought on ease of understanding and completing the measure. Feedback indicated that the measure and the time required to complete it (between three and five minutes) were largely acceptable but that some questions were convoluted and difficult to understand. Piloting also established that our original response set was inappropriate for some questions. For example the question 'I have learnt something about other people's cultures' was difficult to answer in relation to the frequency scale used. Questions that had proved difficult to understand were rewritten with the assistance of the service user research group. Although the commonly used 'strongly agree' to 'strongly disagree' response set made more sense in relation to our questions, our concurrent pilot of an empowerment measure indicated that participants also found this difficult to use. For both measures we therefore adapted the responses to a more user-friendly set (not at all, not particularly, yes a bit, yes definitely). This had most of the values of the Likert scale and made sense over the whole questionnaire.

Respondents were asked to indicate the extent to which each statement had applied to them over the previous 3 months and this proved acceptable.

\section{Field testing}

The revised measure was included in a questionnaire used to obtain baseline data for an outcomes study carried out to determine changes in mental health, empowerment and social inclusion amongst participants of community arts projects (Phase 2 of the research). Projects that had responded to the Phase 1 survey were invited to assist with recruiting participants to the outcomes study by giving new participants at their project an information sheet and letter of invitation from the research team. Those 
who wished to take part were then given the questionnaire with a Freepost envelope for return to the research team.

The alpha statistic was used to assess internal consistency based on item correlations with each scale and with the measure as a whole. Because the field test was carried out within a restricted timescale as part of an ongoing study it has not yet been possible to assess test-retest reliability.

\section{Results}

\section{Concept development}

Table 1 shows the original items included in the baseline questionnaire and their relationship to concepts derived from the literature review.

Table 1 about here

\section{Field test}

Twenty two arts and mental health projects located across England recruited a total of 90 participants to the baseline study. Two participants had completed less than a third of the questionnaire and had to be excluded from the analysis. Of the 88 participants included, 60 (68\%) were women. Participants ranged in age from under $26(6 \%)$ to 55 or older (23\%). Most (56\%) were aged between 36 and 54. Eighty six percent described themselves as from White British or other white ethnic backgrounds.

The majority of participants indicated that they were experiencing depression $(31 \%)$ or depression combined with anxiety $(28 \%)$. Eight people $(10 \%)$ indicated that they had a diagnosis of schizophrenia and $13(16 \%)$ a diagnosis of bipolar disorder. Other mental health difficulties such as eating disorders, agoraphobia or obsessive compulsive disorder were described by $15 \%$. The mental health measure included in the questionnaire (Clinical Outcomes in Routine Evaluation, CORE systems group, 1998) allowed us to estimate the proportion of our sample with a clinically significant mental health difficulty: Fifty seven people (65\%) scored over the clinically significant threshold. Based on their answers to questions about service use, the majority $(61 \%)$ had contact with a specific mental health service (counsellor, psychiatrist, mental health team or social services) at least once a week, with a further $21 \%$ using these services between once a week and once a month.

In scoring the social inclusion measure, we coded responses using a numerical scale of $1-4$ (4 most positive, 1 least positive). We used mean scores for each subscale and mean scores over the whole measures for the analyses described below. There were a number of missing values for two of the 88 participants, and they were excluded. For missing values on the empowerment and social inclusion measure we interpolated using linear trends. There were no cases with systematic patterns and no significant residual effects. No more than six values across the whole population were inserted across the scale scores for each measure. For the CORE, we followed the recommended procedure of restricting missing raw data to three values in any dimension when calculating mean scores. 
Table 2 shows the results for internal consistency. These indicate that some of the original item categories derived from the literature did not constitute consistent scales, but that 16 of the 22 items included in the measure could be grouped within three scales measuring social isolation, social relations and social acceptance. Item correlations for the measure as a whole were good $($ Alpha $=0.85)$ and the constituent scales of social isolation (alpha $=0.76$ ), social acceptance $(0.76)$ and social relations (0.70) were acceptable. Items 2 and 22 shown in Table 1 correlated well with two of the three scales and were therefore included in both, following Gallie \& Paugham (2002). Items 1, 13 and 15 shown in Table 1 had poor correlations with all three scales and with the measure as a whole and were therefore excluded. For item 1, however, most of the confusion was due to the reference to family relations. We retested the scale with an item derived from the CORE that was similar in content for isolation but without the family association. This item correlated well and was retained. Items 14, 16 and 19 shown in Table 1 did not correlate well with any of the three scales but had good correlations with the measure as a whole. These items were therefore excluded from the scales but included as separate items within the measure because the literature indicated that they constituted important elements of social inclusion.

\section{Table 2. about here}

Table 3 shows a Pearson correlation analysis used to contrast the means of the three subscales in order to determine unidimensionality. It can be seen that all the scales correlate strongly with each other and very strongly with the total concept. Social isolation is the strongest dimension and social acceptance the least well fitting. Each dimension seems to tap a different sub-concept but they all correlate very highly with the overarching model indicating that the entire scale is best seen as a single measure.

Table 3 about here

Table 4 shows the relationships between scores on the empowerment measure adapted for the study (Schafer, 2002) and the CORE, both administered at the same time. There is a highly significant correlation between all three measures. Correlation with the CORE results is negative because higher scores on the CORE are indicative of more problems whereas higher scores for empowerment and social inclusion are indicative of fewer problems. The correlations at 0.6 do not exactly relate to each other, indicating that each measures a different dimension of the same construct. Scores correlated well and thus demonstrate predictive validity. Empowerment correlated best with both social inclusion and mental health as reflected by the CORE. Social inclusion correlated least well with the CORE. The indications are that poor mental health is generally associated with low levels of social inclusion.

Table 4 about here

\section{Discussion}


Although promoting the social inclusion of people with mental health problems continues to be a key focus of UK government policy (Social Exclusion Task Force, 2006), conceptualisation and measurement of the construct is at an early stage. The measure described here represents one contribution made in the specific context of a study aimed at developing the evidence base for participation in arts and mental health projects.

The tests it has been possible to carry out to date indicate that the measure is reasonably straightforward to complete and measures relevant concepts in areas where interventions are likely to have impact. The three scales constructed as a result of field testing have acceptable levels of item correlation and the whole scale consistency score of 0.85 indicates a good relation to elements of social inclusion in community networks. However, the scales relate more to inclusion at the individual level in close social networks than to inclusion at the group level in terms of economic inclusion and equity of access (Walker \& Walker, 1997).

Correlations with scores on the CORE and empowerment measures indicate that the measure has reasonable predictive validity for the population with which it was tested. The three scales also relate well to scales measuring social devaluation, inclusion and isolation included in previous surveys with other populations (Gallie \& Paugham, 2002), indicating that at least some of the essential elements of social inclusion appear. Nevertheless, construct validity cannot be claimed on the basis of the tests to date, because it is questionable whether the whole construct of social inclusion is fully represented by the three dimensions included in the measure. In particular, the measure relates to subjective aspects of people's lives and does not include more objective indicators such as levels of income and employment. However, our literature review indicated that social inclusion and social exclusion are best viewed as separate dimensions rather than as opposite ends of a continuum. Whereas exclusion can be seen as stemming from structural barriers to participation in society, inclusion implies a radial shift in attitudes to marginalised groups. For example, someone with mental health problems may obtain paid work and thus be seen to have attained a productive social role, but if they are ostracised in the workplace this hardly constitutes inclusion. It is therefore arguable that the more objective indicators relate to exclusion rather than to inclusion, which can only be measured subjectively. This clearly remains an issue for further research and theoretical debate.

Other limitations include the need to establish test-retest reliability and a normative profile for this population, neither of which were possible in the context of this study. The measure was also developed for use with a specific population of arts project participants, the majority of whom were women, in their middle years, from White ethnic backgrounds and experiencing depression. The context specific wording of some items will require adaptation for use with other populations and testing of internal consistency will also require further replication. 
Development of a measure of social inclusion

\section{References}

Bates P. (2005) Review of measures of social inclusion. Ipswich: National Development Team.

Colgan S., Bridges K. \& Faragher B. (1991) A tentative START to community care. Psychiatric Bulletin 15, 596 - 598

Core System Group (1998) CORE System (Information management) Handbook. Leeds: Core System Group, University of Leeds.

Everitt A. \& Hamilton R. (2003) Art, Health \& Well-being - An Evaluation of Five Community Arts in Health Projects. Durham: Centre for Arts and Humanities in Health and Medicine, Durham University.

Hacking S, Secker J., Kent L., Shenton J. \& Spandler H (2006) Mental health and arts participation: the state of the art in England. Journal of the Royal Society of Health Promotion 126 (3), 121-127.

Health Development Agency (2000) Arts for Health: A Review of Good Practice in Community-Based Arts Projects and interventions which impact on health and wellbeing. London: Health Development Agency.

Huxley P. (1997) Arts on Prescription. Stockport: Stockport NHS Trust.

Gallie, D. \& Paugham, S. (2002) Social Precarity and Social Integration. Brussels: European Commission, Directorate General, Eurobarometer 56.1.

Matarasso, F, (1997) Use or Ornament? The Social Impact of Participation in the Arts Stroud: Comedia.

McKenzie K., Whitely R. and Weich S. (2002) Social capital and mental health. British Journal of Psychiatry 181 (4), 280 - 283

ODPM (2004) Mental Health and Social Exclusion. London: Office of the Deputy Prime Minister.

Putnam R. (1995) Bowling Alone: America's declining social capital. Journal of Democracy 6 (1), 65-78.

Schafer T. (2000) Empowerment: towards a participatory model for the evaluation of the empowering therapeutic environment. Mental Health Care 3 (7) 233 - 237.

Social Exclusion Task Force (2006) Reaching Out: An Action Plan on Social Exclusion. London: Cabinet Office.

Stickley T. \& Shaw R. (2006) Evaluating Social inclusion. Mental Health Today 9 (10), $14-20$. 
Development of a measure of social inclusion

Walker A. and Walker C. (1997) Britain Divided: the Growth of Social Exclusion in the 1980s and 1990s, London: Child Poverty Action Group. 
Table 1 Baseline social inclusion items

\begin{tabular}{|c|c|c|}
\hline & Item & $\begin{array}{l}\text { Concept } \\
\text { derivation }\end{array}$ \\
\hline 1 & *I have felt isolated from my family & \multirow{3}{*}{$\begin{array}{l}\text { Related to social } \\
\text { capital (bonding) }\end{array}$} \\
\hline 2 & *I have friends I see or talk to every week & \\
\hline 3 & $\begin{array}{l}\text { *My social life has been mainly related to mental health } \\
\text { services or people who use mental health services }\end{array}$ & \\
\hline 4 & $\begin{array}{l}\text { *I have been involved in a group, club or organisation } \\
\text { that is not just for people who use mental health } \\
\text { services (not including your arts project) }\end{array}$ & \multirow[t]{3}{*}{$\begin{array}{l}\text { Related to social } \\
\text { capital (bridging) }\end{array}$} \\
\hline 5 & I have learnt something about other people's cultures & \\
\hline 6 & I have been to new places (other than your arts project) & \\
\hline 7 & *I have felt accepted by my friends & \multirow[t]{5}{*}{ Social acceptance } \\
\hline 8 & *I have felt accepted by my family & \\
\hline 9 & *I have felt accepted by my neighbours & \\
\hline 10 & $\begin{array}{l}\text { *I have felt that some people look down on me because } \\
\text { of my mental health needs }\end{array}$ & \\
\hline 11 & I have felt what I do is valued by others & \\
\hline 12 & $\begin{array}{l}\text { *I have felt it was unsafe to walk alone in my } \\
\text { neighbourhood in daylight }\end{array}$ & \multirow[t]{2}{*}{$\begin{array}{l}\text { Neighbourhood } \\
\text { cohesion }\end{array}$} \\
\hline 13 & I have had problems with my neighbours & \\
\hline 14 & $\begin{array}{l}\text { I have felt insecure about where I live (for example } \\
\text { afraid I might be evicted) }\end{array}$ & \multirow[t]{2}{*}{$\begin{array}{l}\text { Stability of } \\
\text { housing tenure }\end{array}$} \\
\hline 15 & *I have been behind with my rent / mortgage & \\
\hline 16 & $\begin{array}{l}\text { *I have done a sport, game or physical activity (not just } \\
\text { walking to get somewhere) }\end{array}$ & \multirow{3}{*}{$\begin{array}{l}\text { Engagement in } \\
\text { leisure \& cultural } \\
\text { activities }\end{array}$} \\
\hline 17 & $\begin{array}{l}\text { *I have been out socially with friends (for example to } \\
\text { the cinema, restaurants, pubs, clubs) }\end{array}$ & \\
\hline 18 & $\begin{array}{l}\text { I have done some cultural activities (for example gone } \\
\text { to a library, museum, gallery, theatre or concert) }\end{array}$ & \\
\hline 19 & $\begin{array}{l}\text { *I have helped out at a charity or local group (other than } \\
\text { a mental health group) }\end{array}$ & \multirow[t]{4}{*}{ Citizenship } \\
\hline 20 & I have felt clear about my rights & \\
\hline 21 & $\begin{array}{l}\text { I have felt free to express my beliefs (for example } \\
\text { political or religious beliefs) }\end{array}$ & \\
\hline 22 & *I have felt that I am playing a useful part in society & \\
\hline
\end{tabular}

* Adapted from national surveys 
Development of a measure of social inclusion

Table 2 Social inclusion measure

\begin{tabular}{|l|c|c|c|}
\hline Social Inclusion Scale & $\begin{array}{c}\text { Internal } \\
\text { consistency } \\
\text { item alpha }\end{array}$ & $\begin{array}{c}\text { Total } \\
\text { scale } \\
\text { alpha }\end{array}$ & $\begin{array}{c}\text { Subscale } \\
\text { (ALPHA) }\end{array}$ \\
\hline I have felt terribly alone and isolated & 0.54 & 0.49 & Social \\
I have felt accepted by my friends & 0.56 & 0.57 & $\begin{array}{c}\text { isolation } \\
\text { I have been out socially with friends }\end{array}$ \\
I have felt I am playing a useful part in society & 0.45 & 0.41 & (0.76) \\
I have friends I see or talk to every week & 0.60 & 0.63 & \\
\hline I have felt I am playing a useful part in society & 0.59 & X & \\
I have felt what I do is valued by others & 0.57 & 0.95 & \\
My social life has been mainly related to MH or people who & & & \\
use MH services & 0.35 & 0.36 & \\
I have been to new places & 0.52 & 0.47 & Social \\
I have learnt something about other cultures & 0.32 & 0.29 & relations \\
I have been involved in a group not just for MH & 0.34 & 0.41 & (0.76) \\
I have done some cultural activity & 0.51 & 0.57 & \\
I have felt some people look down on me because of my MH & & & \\
needs & 0.39 & 0.46 & \\
I have felt unsafe to walk alone in my neighbourhood in & & & \\
daylight & 0.32 & 0.34 & \\
\hline I have felt accepted by neighbours & 0.47 & 0.32 & \\
I have friends I see or talk to every week & 0.41 & X & Social \\
I have felt accepted by my family & 0.43 & 0.33 & acceptance \\
I have felt clear about my rights & 0.46 & 0.46 & (0.70) \\
I have felt free to express my beliefs & 0.49 & 0.51 & \\
\hline I have felt insecure about where I live & Excluded & 0.32 & Not in \\
I have done a sport, game or physical activity & Excluded & 0.35 & subscales \\
I have helped out at a charity or local group & Excluded & 0.43 & \\
\hline Internal consistency for whole scale & & $\mathbf{0 . 8 5}$ & \\
Chronbach's Alpha & & & \\
\hline
\end{tabular}


Development of a measure of social inclusion

Table 3 Pearson Correlation amongst subscales and with whole scale

\begin{tabular}{|l|r|r|r|}
\hline $\begin{array}{l}\text { Pearson Correlation } \\
\text { Coefficient among subscales }\end{array}$ & \multicolumn{1}{|l|}{$\begin{array}{l}\text { Social } \\
\text { Isolation }\end{array}$} & \multicolumn{1}{l|}{$\begin{array}{l}\text { Social } \\
\text { Relations }\end{array}$} & \multicolumn{1}{l|}{ Social Acceptance } \\
\hline Social Isolation & 1.0 & $0.70^{* * *}$ & $0.70^{* * *}$ \\
\hline Social Relations & $0.66^{* * *}$ & 1 & $0.52^{* * *}$ \\
\hline Social Acceptance & $0.69^{* * *}$ & $0.52^{* * *}$ & 1 \\
\hline Inclusion all scales & $0.85^{* * *}$ & $0.91^{* * *}$ & $0.78^{* * *}$ \\
\hline
\end{tabular}

$* * *$ Correlation is significant at the 0.001 level (2-tailed). All N=86

Table 4 Pearson Correlation between mean scores for Social Inclusion, Empowerment and CORE (symptoms)

\begin{tabular}{|l|r|r|r|r|}
\hline & Mean (SD) & Empowerment & CORE & \multicolumn{1}{c|}{ Inclusion } \\
\hline Empowerment & $6.23(1.76)$ & 1 & $-.653 * * *$ & $-.615 * * *$ \\
\hline CORE & $1.60(0.70)$ & $-.653 * * *$ & 1 & $.582 * * *$ \\
\hline Inclusion & $2.40(0.61)$ & $-.615 * * *$ & $.582 * * *$ & 1 \\
\hline
\end{tabular}

*** Correlation is significant at the 0.001 level (2-tailed). All N=85 